\title{
Effectiveness Comparison Between Fecal Immunochemical Test And Colonoscopy As A Tool For Early Detection Of Colorectal Cancer
}

\author{
Yossi Andila*, Reno Rudiman*, Andriana Purnama* \\ *Department of Surgery, Dr. Hasan Sadikin General Hospital-Padjadjaran \\ University, Bandung, West Java, Indonesia \\ DOI: 10.29322/IJSRP.11.09.2021.p11722 \\ http://dx.doi.org/10.29322/IJSRP.11.09.2021.p11722
}

\begin{abstract}
Cancer is a disease that causes a lot of misery and death in humans. According to data from the WHO (World Health Organization) in 2015, there were 8.8 million deaths from cancer and colorectal cancer took third place with 774,000 deaths. One of the colorectal cancer preventions can be done through early detection in the population, especially in high-risk groups and in groups with non-specific symptoms. In general, two types of early detection tests are available: stool-based testing and structural tests. Fecal Immunochemical Test (FIT) is one type of examination that is included in the stool based test. While the examinations that are classified as structural tests include colonoscopy, CT colonography and flexible sigmoidoscopy. This study aims to determine differences in the effectiveness of the FIT against colonoscopy as an early detection tool for colorectal cancer. This study was a diagnostic test study which was an analytical observational study using a cross-sectional design to compare early detection of FIT with colonoscopy in colorectal cancer patients at Dr. Hasan Sadikin Bandung General Hospital. Analysis of diagnostic test data was carried out to obtain sensitivity, specificity, positive and negative predictive value and bivariate analysis using Man Whitney test. 38 patients were included in this study, with 27 cancer and 11 non-cancer patients. Based on the results of data analysis, the sensitivity level of the FIT was $81.5 \%$, the specificity level was $72.7 \%$. There was no significant difference in the effectiveness of the FIT against Colonoscopy as a screening tool for colorectal cancer.
\end{abstract}

Index Terms- Cancer, Colorectal Cancer, Colonoscopy, Comparative Studies, Fecal Immunochemical Test

\section{INTRODUCTION}

$\mathrm{C}^{\mathrm{s}}$ olorectal cancer is a malignancy originating from the large intestine, consisting of the colon (the longest part of the large intestine) and/or the rectum (the last small part of the large intestine before the anus). According to the American Cancer Society, colorectal cancer is the third most common cancer and the third leading cause of cancer death in men and women in the United States. Based on the 2018 GLOBOCAN survey (Global Burden of Cancer Study), the incidence of colorectal cancer worldwide ranks third (1360 out of 100,000 population) and ranks fourth as a cause of death (694 out of 100,000 population). ${ }^{1}$

Colorectal cancer is generally more common in elderly individuals and is very rare in children. Suspicion of colorectal cancer can be seen based on the symptoms experienced by the patient. Symptoms that often arise in someone suffering from colorectal cancer are bleeding in the lower gastrointestinal tract which is characterized by bloody discharge during defecation, an increase in the amount of feces produced and often accompanied by diarrhea that lasts for more than 6 weeks. In addition, other symptoms are signs of blockage in the intestines and the occurrence of weight loss for no apparent reason. ${ }^{1,2}$ Colorectal cancer/carcinoma can be diagnosed by definitive biopsy, including fine needle aspiration biopsy (FNAB), core biopsy, or subtotal removal of a single node. Microscopic examination by a pathologist is necessary to identify the molecular, cellular or architectural characteristics of the epithelial tissue. Carcinoma cancer cell structure has a large nucleus size, round monotone and has abundant cytoplasm. Microscopically, an adenocarcinoma can be seen with various glandular arrangements and cell types, some containing excessive mucin in the cytoplasm to be called signet-ring cells or forming so much mucin that it is called mucoid carcinoma. ${ }^{1,3}$

Prompt diagnosis and management helps reduce the risk of dying from colorectal cancer. Appropriate diagnosis can be assisted by early detection of patients suspected of having colorectal cancer symptoms. Apart from being the first step in establishing a diagnosis, early detection also aims to remove the lesion pre-cancer and specifically detects disease at an early stage so that curative therapy can be performed. ${ }^{4}$ Early detection of colorectal cancer is primarily indicated in patients with the following conditions: Individuals with a history of adenomatous polyps. Individuals with a history of colorectal cancer curative resection. Individuals with first-degree relatives with a history of colorectal cancer or colorectal adenoma (recommendations differ based on family age at diagnosis). Individuals with a long history of inflammatory bowel disease. Individuals with a diagnosis or suspicion of hereditary nonpolyposis colorectal cancer (HNPCC) syndrome or Lynch syndrome or familial adenomatous polyposis (FAP). ${ }^{5}$

In general, there are 2 types of early detection that can be done, namely Stool Based Test and Structural Exams. In the Stool Based examination, the sample used is feces by looking at several components that may appear. This test is considered non-invasive, 
easy to do but should be done more often. In addition, this examination will still require confirmation through examination visual (structural) exams if abnormal results are found ${ }^{6}$ One of the best ways to check for cancer is to look for blood in the stool. This is because in cases of colorectal polyps or colorectal cancer there will be neovascularization that is fragile and will be very easily damaged when feces pass through these lesions. But often the resulting blood cannot be seen with the naked eye. Examination of fecal immunochemical test (FIT) is one method of assessing the presence or absence of blood in the stool. This check is easier to do but must be done every year. ${ }^{6}$

The FIT examination has several differences compared to the Fecal Occult Blood Test (FOBT) which is also included in the Stool Based Test. In the FIT examination, it is not necessary to do a special diet, or treatment must be stopped first because vitamins and food will not affect the results of the examination. This examination is also less responsive to upper gastrointestinal bleeding so the possibility of bias due to other bleeding is lower. ${ }^{7}$ The sampling procedure of the FIT can also be performed independently by the patient. Patients will be provided with the necessary equipment for sampling such as test kits, test cards/tubes, long brushes/collecting devices, and waste bags. ${ }^{8}$

Meanwhile, colonoscopy, which is one of the modalities of Structural Exams, can be performed by looking at the colon and rectum using a colonoscope. A colonoscope is a flexible tube about the size of a finger with a video camera at the end. The colonoscope is inserted through the anus into the rectum and colon. This colonoscope is also accompanied by an instrument to take samples if needed. ${ }^{7}$ Based on its advantages, FIT has advantages in the form of no risk of trauma to the colon because the examination is not invasive, no special preparation is needed, the sampling process is easier to do and can be done at home, and cheaper. However, there are some drawbacks to FIT, such as not being able to detect some types of polyps and cancer, it can give results false positive, it must be done every year and a colonoscopy should still be done if abnormal results are found. ${ }^{6,18}$ On the other hand, colonoscopy is still considered to be the most accurate test for early detection and prevention of colorectal cancer but also has constraints in terms of cost and patient convenience. Both of these modalities have their respective advantages and disadvantages so that researchers are interested in comparing the effectiveness of the Fecal Immunochemical Test (FIT) against colonoscopy. as an early detection tool for colorectal cancer.

\section{METHODS}

This study was conducted at Dr. Hasan Sadikin Bandung General Hospital during the period January 2021 - April 2021 with the subject were adult colorectal cancer patients aged more than 18 years who came to the Digestive Surgery Department of Dr. Hasan Sadikin Bandung General Hospital which meets the inclusion criteria and does not include the exclusion criteria. This study consisted of independent variables in the form of Fecal Immunochemical Test (FIT) and colonoscopy as an early detection tool and the effectiveness of each therapy as the dependent variable. This research procedure was approved by the Health Research Ethics Committee No.LB.02.01/X.6.5/300/2021, Universitas Padjadjaran Bandung.

This study was an observational analytic diagnostic test study using a cross-sectional design that aimed to compare the effectiveness of the Fecal Immunochemical Test (FIT) against colonoscopy. as an early detection tool for colorectal cancer.

This study was conducted on patients who have symptoms of colorectal cancer with an affordable population including patients undergoing examination and treatment at the Digestive Surgery Department of Dr. Hasan Sadikin Bandung General Hospital period January 2021 - April 2021. Research subjects must be patients with suspected colorectal cancer and a high risk of colorectal cancer, aged more than 18 years and willing to participate in the study for a predetermined period of time after has been informed consent obtained. The exclusion criteria in this study were patients who had undergone surgery and patients who were undergoing or had completed chemotherapy.

The minimum sample size was determined based on the sample size formula for diagnostic tests with a $95 \%$ confidence interval. Meanwhile, colonoscopy's sensitivity method expected was $90 \%$. So that the minimum number of samples needed was 35 patients, added $10 \%$ to avoid sample shortages due to drop out so that it becomes 38 patients. The sampling technique was carried out by consecutive sampling, namely based on the order of arrival of patients who met the inclusion criteria until the minimum sample size was met. Determination of the sample starts from the selection of members of the population against the inclusion and exclusion criteria. Where the selected sample was a sample that can be reached by researchers and has met the inclusion criteria and exclusion criteria.

All research subjects were examined by anamnesis related to colorectal cancer. Physical examination was also assessed to help diagnose colorectal cancer. Subsequent research subjects underwent FIT examination and colonoscopy to diagnose suspected colorectal cancer. The analysis carried out was a diagnostic test to obtain sensitivity, specificity, positive predictive value (PPV), and negative predictive value (NPC). Furthermore, bivariate analysis was carried out by using a different test using chi square for comparison of colorectal cancer diagnosis using FIT with Colonoscopy. And testing the hypothesis using the Mann Whitney test. The results of statistical tests were said to be meaningful if the p value was less than 0.05 . The data obtained were recorded in a special form and then processed through the SPSS version 21.0 program for Windows.

\section{RESULTS}

During the period January 2021 - April 2021, 38 patients with symptoms resembling colorectal cancer were examined and treated at the Digestive Surgery Department, Dr. Hasan Sadikin Bandung General Hospital, with details as many as 27 people were 
colorectal cancer patients and 11 patients who were not colorectal cancer. The following table was the result of the identification of the research subject characteristics totaling 38 patients.

Table 1 Characteristics of Research Subjects

\begin{tabular}{|c|c|c|c|}
\hline Variable & & Total & Percentage \\
\hline \multirow[t]{6}{*}{ Age } & 17-25 Years & 1 & $2.6 \%$ \\
\hline & 26-35 Years & 5 & $13.2 \%$ \\
\hline & 36-45 Years & 4 & $10.5 \%$ \\
\hline & 46-55 Years & 9 & $23.7 \%$ \\
\hline & 56-65 Years & 12 & $31.6 \%$ \\
\hline & $>65$ Years & 7 & $18.4 \%$ \\
\hline \multirow[t]{2}{*}{ Gender } & Male & 18 & $47.4 \%$ \\
\hline & Female & 20 & $52.6 \%$ \\
\hline \multirow[t]{2}{*}{ Comorbid } & Yes & 29 & $76.3 \%$ \\
\hline & No & 9 & $23.7 \%$ \\
\hline \multirow[t]{6}{*}{ Risk Factors } & Physical Activity, Diet & 1 & $2.6 \%$ \\
\hline & Diet & 5 & $13.2 \%$ \\
\hline & Hereditary Factors & 1 & $2.6 \%$ \\
\hline & Hereditary Factors, Diet & 1 & $2.6 \%$ \\
\hline & Smoking & 3 & $7.9 \%$ \\
\hline & Smoking and Age & 1 & $2.6 \%$ \\
\hline
\end{tabular}




\begin{tabular}{|c|c|c|}
\hline Smoking, Diet & 2 & $5.3 \%$ \\
\hline Age & 6 & $15.8 \%$ \\
\hline Age, Physical activity & 4 & $10.5 \%$ \\
\hline Age, Diet & 5 & $13.2 \%$ \\
\hline Age, Hereditary factors, diet & 1 & $2.6 \%$ \\
\hline Age, smoking & 3 & $7.9 \%$ \\
\hline Age, Smoking & 4 & $10.5 \%$ \\
\hline Age, smoking, Diet & 1 & $2.6 \%$ \\
\hline
\end{tabular}

Based on table 1 it can be seen that the age of the majority of research subjects was in the range of 56-65 years with a percentage of $31.6 \%$ or 12 patients. Then the second place, namely the age range of $46-55$ years, totaling 9 people (23.7\%). While the lowest age was $17-25$ years only 1 person $(2.6 \%)$. When viewed by gender, $52.6 \%$ of patients were female and $47.4 \%$ were male. A total of 29 people $(76.3 \%$ ) had comorbidities and only $23.7 \%$ had no comorbidities. There were several risk factors in patients, include the most combination of age, physical activity, diet and smoking. These factors have a higher percentage when compared to other risk factors.

Analysis of hypothesis testing was carried out using the Mann Whitney test comparison. Then tested for sensitivity, specificity, positive predictive value (PPV), and negative predictive value (NPV). The following was a recapitulation of the hypothesis testing result.

Table 2 Sensitivity, Specificity, PPV and NPV of FIT and Colonoscopy Examination

\begin{tabular}{llll}
\hline \multirow{2}{*}{ Colonoscopy } & \multicolumn{2}{c}{ FIT } \\
\cline { 2 - 3 } Positive & Positive & Negative & 27 \\
Negative & 22 & 5 & 11 \\
\hline Total & 3 & 8 & 38 \\
\hline
\end{tabular}




$\begin{array}{llllll}81.5 \% & 72.7 \% & 88.0 \% & 61.5 \% & 0.514 & 0.624\end{array}$

\section{*) p-value mann whitney test}

Based on the recapitulation of the results in table 2, it can be seen that the p-value of the Mann Whitney test showed 0.624. Because the p-value > 0.05 means that there was no statistically significant difference in the effectiveness of the Fecal Immunochemical Test on Colonoscopy as a screening tool for colorectal cancer. In other words, the results of the Fecal Immunochemical Test (FIT) were almost the same as the results on Colonoscopy. The sensitivity level of the Fecal Immunochemical Test (FIT) was $81.5 \%$, the specificity level was $72.7 \%$, the PPV value was $88 \%$ and the NPV value was $61.5 \%$. Thus, the research hypothesis can be accepted where there was no significant difference between the effectiveness of the Fecal Immunochemical Test against Colonoscopy as a screening tool for colorectal cancer.

\section{DISCUSSION}

FIT and colonoscopy were two modalities that can be used as methods of early detection of colorectal cancer. FIT has advantages in the form of no risk of trauma to the colon because the examination is not invasive, no special preparation is needed, the sampling process is easier to do and can be done at home, cheaper. In addition, a study conducted by Segnan et al. in 2007 has shown that semiquantitative FIT was more accurate than the guaiac test for detecting advanced colorectal cancer and adenoma, and this new test was now recommended as the fecal occult blood test first choice in the early detection of colorectal cancer. However, there were some limitations from FIT, such as not being able to detect certain types of polyps and cancer, it can give false positive results, it must be done every year and a colonoscopy should still be done if abnormal results are found. ${ }^{7}$

Colonoscopy on the other hand was still the gold standard in detecting colorectal cancer and was recommended as the first line for early detection because its accuracy was still higher than other tests. However, colonoscopy still has several disadvantages, including costs that are not cheap and are more invasive which in turn will reduce patient comfort and patient compliance in carrying out the examination. ${ }^{9}$ The balance between invasive and non-invasive examinations was considered to have an important role in increasing patient compliance for early detection for further diagnosis. ${ }^{10,11}$

Although both can be used as an early detection tool and was quite sensitive in detecting colorectal cancer lesions, but fecal immunochemical test has a limited function in detecting pre-cancerous lesions compared to colonoscopy. However, if FIT is as effective as colonoscopy the use of this test as an early detection tool can reduce the cost burden significantly. ${ }^{12,13}$

The results of this study were similar to those of Enrique et al. who also described that there was no significant difference between colonoscopy and FIT. ${ }^{5}$ Meanwhile differing results obtained in studies conducted Amanda, et al that FIT can reduce the use of colonoscopy to $71 \%$, but can miss cases of colorectal cancer up to $30-40 \%$ of cases. ${ }^{14}$

In a study conducted by Zorzi et al. Colonoscopy was known to more accurately describe the type of cancer and the stage of cancer that occurs in patients. The results of the study stated that there were 3.9\% of patients suffering from colorectal cancer, of which $24.8 \%$ had a high risk of developing an adenoma and $18.7 \%$ a low risk of developing an adenoma. As for the stage of colorectal cancer can be detected in $64.8 \%$ of patients, and obtained patients with the condition of stage I as many as $48.6 \%$ and stage II as many as $34.5 \%$. Limitations of this study was the site of neoplasms that are not described further even though the actual location of the neoplasm may affect the sensitivity and specificity of both the early detection tool. Colonoscopy and FIT were known to be less effective in detecting lesions located in the proximal colon than in the distal colon. ${ }^{2,15}$

\section{CONCLUSION}

From the results of research on the comparison of the effectiveness of the Fecal Immunochemical Test (FIT) against Colonoscopy as an early detection tool for colorectal cancer, it was known that there was no significant difference between the effectiveness of the Fecal Immunochemical Test against Colonoscopy as a screening tool for colorectal cancer. This study can be further developed, with more samples, more variables such as the type of lesion, whether pre-cancerous or cancerous, described in more detail and then the location of the neoplasm can also be added because the sensitivity and specificity of FIT and colonoscopy are influenced by other factors. that factor.

\section{REFERENCES}

[1] Basir I, Rudiman R, Lusikoy R, Lukman K, Saditya W, Jeo WS, et al. 2016. Panduan Penatalaksanaan Kanker kolorektal. Kementerian Kesehatan Republik Indonesia. 2016.76-81.

[2] Vieito NP, Zarraquiños S, Cubiella J. High-risk symptoms and quantitative faecal immunochemical test accuracy: systematic review and meta-analysis. World journal of gastroenterology. 2019;25(19):2383-2401.

[3] Prentice A, Marshall S, Vance M, Choglay S, Von Wagner C, Kerrison R. Colorectal cancer screening and the role of community pharmacy. The Pharmaceutical Journal. 2019;302(1):1-11. 
[4] D'Souza N, Georgiou Delisle T, Chen M, Benton S, Abulafi M. Faecal immunochemical test is superior to symptoms in predicting pathology in patients with suspected colorectal cancer symptoms referred on a 2WW pathway: a diagnostic accuracy study. Gut. 2021;70(6):1130-1138.

[5] van Turenhout ST, Oort FA, van der Hulst RWM, Visscher AP, sive Droste JS, Scholten P, et al. Prospective cross-sectional study on faecal immunochemical tests: sex specific cut-off values to obtain equal sensitivity for colorectal cancer? BMC Gastroenterol. 2014;14:217.

[6] Siegel RL, Miller KD, Jemal A. Cancer statistics, 2016. CA Cancer J Clin. 2016;66(1):7-30.

[7] Helsingen LM, Vandvik PO, Jodal HC, Agoritsas T, Lytvyn L, Anderson JC, et al. Colorectal cancer screening with faecal immunochemical testing, sigmoidoscopy or colonoscopy: a clinical practice guideline. BMJ. 2019(2);367.

[8] Nicholson BD, Thompson M, Price CP, Heneghan C, Plüddemann A. Home-use faecal immunochemical testing: primary care diagnostic technology update. Br J Gen Pract J R Coll Gen Pract. 2015;65(632):156-158.

[9] Zorzi M, Hassan C, Capodaglio G, Baracco M, Antonelli G, Bovo E, eta 1. Colonoscopy later than 270 days in a fecal immunochemical test-based population screening program is associated with higher prevalence of colorectal cancer. Endoscopy. 2020;52(10):871-6.

[10] Li SJ, Sharples LD, Benton SC, Blyuss O, Mathews C, Sasieni P, et al. Faecal immunochemical testing in bowel cancer screening: Estimating outcomes for different diagnostic policies. Journal of Medical Screening. 2020;12(0969141320980501):1-19.

[11] Atkin W, Dadswell E, Wooldrage K, Kralj-Hans I, von Wagner C, Edwards R, et al. Computed tomographic colonography versus colonoscopy for investigation of patients with symptoms suggestive of colorectal cancer (SIGGAR): a multicentre randomised trial. The Lancet. 2013;381(9873):1194-202.

[12] Moss S, Mathews C, Day TJ, Smith S, Seaman HE, Snowball J, et al. Increased uptake and improved outcomes of bowel cancer screening with a faecal immunochemical test: results from a pilot study within the national screening programme in England. Gut. 2017;66(9):1631-44.

[13] 1Steele RJ, McDonald PJ, Digby J, Brownlee L, Strachan JA, Libby G, et al. Clinical outcomes using a faecal immunochemical test for haemoglobin as a first-line test in a national programme constrained by colonoscopy capacity. United European gastroenterology journal. 2013;1(3):198-205.

[14] Quintero E, Castells A, Bujanda L, Cubiella J, Salas D, Lanas Á, et al. Colonoscopy versus fecal immunochemical testing in colorectal-cancer screening. New England Journal of Medicine. 2012;366(8):697-706.

[15] Khan AA, Klimovskij M, Harshen R. Accuracy of faecal immunochemical testing in patients with symptomatic colorectal cancer. BJS Open. 2020;4(6):11801188.

\section{AUTHORS}

First author- Yossi Andila, Department of Surgery, Dr. Hasan Sadikin General Hospital-Padjadjaran

University, Bandung, West Java, Indonesia. Email: yossiandilla@gmail.com

Second author- Reno Rudiman, Department of Surgery, Dr. Hasan Sadikin General Hospital-Padjadjaran

University, Bandung, West Java, Indonesia. Email: Rudiman@ unpad.ac.id

Third author- Andriana Purnama, Department of Surgery, Dr. Hasan Sadikin General Hospital-Padjadjaran

University, Bandung, West Java, Indonesia. Email: apuyboy@yahoo.com

Correspondence Author - Yossi Andila, Department of Surgery, Dr. Hasan Sadikin General Hospital-Padjadjaran

University, Bandung, West Java, Indonesia. Email: yossiandilla@ gmail.com 